\title{
Relationship of Adiposity and Insulin Resistance Mediated by Inflammation in a Group of Overweight and Obese Chilean Adolescents
}

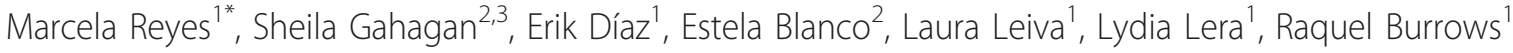

\begin{abstract}
The mild chronic inflammatory state associated with obesity may be an important link between adiposity and insulin resistance (IR). In a sample of 137 overweight and obese Chilean adolescents, we assessed associations between high-sensitivity C-reactive protein (hs-CRP), IR and adiposity; explored sex differences; and evaluated whether hs-CRP mediated the relationship between adiposity and IR. Positive relationships between hs-CRP, IR and 2 measures of adiposity were found. Hs-CRP was associated with waist circumference (WC) in boys and fat mass index (FMI) in girls. Using path analysis, we found that hs-CRP mediated the relationship between adiposity (WC and FMI) and the homeostatic model assessment of insulin resistance (HOMA-IR) $(p<0.05)$ in both sexes. Our novel finding is that inflammation statistically mediated the well described link between increased adiposity and IR.
\end{abstract}

\section{Introduction}

Adolescent obesity is a major public health problem associated with cardiovascular $(\mathrm{CV})$ risk factors including abdominal obesity, insulin resistance (IR), dyslipidemia and hypertension [1,2]. While visceral fat mass is strongly associated with CV risk, causality has not been established [3]. Furthermore, the underlying mechanisms by which excess fat mass (FM) leads to CV risk remain unclear. The mild chronic inflammatory state characterizing obesity may be an important pathophysiologic link between increased adiposity and CV disease [4]. Some investigators have found differences in these associations by sex $[5,6]$. The purpose of this study was to: assess associations between high-sensitivity CRP (hs-CRP), adiposity and the homeostatic model assessment of insulin resistance (HOMA-IR); explore sex differences in these relationships; and evaluate whether inflammation mediated the relationship between adiposity and HOMA-IR in a sample of overweight and obese Chilean adolescents.

\section{Methods}

The sample was drawn from a larger study of nutrition and physical activity among Chilean adolescents (Tanner

\footnotetext{
* Correspondence: mreyes@inta.uchile.cl

'Institute of Nutrition and Food Technology (INTA), University of Chile, Santiago, Chile

Full list of author information is available at the end of the article
}

stage $\geq 2$ ) attending school in Santiago, Chile ( $\mathrm{n}=$ 1,780). Overweight and obese adolescents were invited to participate. Exclusion criteria included: chronic diseases; acute infections; hs-CRP values above $9 \mathrm{mg} / \mathrm{L}$; or any medications. Parents signed informed consent and adolescents signed informed assent. Ethics Board of the Institute of Nutrition and Food Technology, University of Chile (INTA) approved this study. A pediatric endocrinologist performed all anthropometric measurements in duplicate. Each adolescent was measured in the Frankfurt position wearing underwear, without shoes. Body mass index (BMI), sex-and age-specific BMI Zscores, and waist circumference (WC) percentiles were calculated based on the U.S. Centers for Disease Control and Prevention Growth Charts/National Center for Health Statistics standards $[7,8]$. Arterial blood pressure percentiles were classified according the Updated Second Task Force Report recommendations [9]. FM was evaluated using deuterium isotope dilution according to standard methods [10]. We computed FM index (FMI) as follows: fat mass $[\mathrm{kg}] /$ height $\left[\mathrm{m}^{2}\right]$ [11]. After a 12-hr overnight fast, $8 \mathrm{~mL}$ of venous blood were collected. The assays (glucose, insulin, hs-CRP, lipids) were performed at INTA.

Statistical analyses were performed using SPSS (version 17.0, SPSS Inc., Chicago, IL, US) and SAS software (version 9.2, SAS Institute, Cary, NC, US). Bivariate 
Table 1 Adiposity and CV Risk, by sex†

\begin{tabular}{lcc}
\hline & Male & Female \\
& $\mathbf{n}=\mathbf{7 8}$ & $\mathbf{n}=\mathbf{5 9}$ \\
\hline Age (year) & $14.8(14.7-15.0)$ & $14.7(14.5-14.8)$ \\
BMI (z score) & $2.1(2.1-2.6)$ & $2.3(2.1-2.5)$ \\
WC $(\mathrm{cm})$ & $91.3(90.6-94.8)$ & $90.7(89.5-93.9)$ \\
FMI $\left(\mathrm{kg} / \mathrm{m}^{2}\right)^{*}$ & $8.2(8.1-9.2)$ & $11.1(10.7-11.8)$ \\
HOMA-IR & $2.2(2.3-3.3)$ & $2.1(1.9-2.6)$ \\
hs-CRP $(\mathrm{mg} / \mathrm{L})$ & $0.8(0.9-1.5)$ & $0.7(0.8-1.5)$ \\
\hline
\end{tabular}

† Values are median $(95 \% \mathrm{Cl})$.

* Statistically significant difference between males and females (MannWhitney test).

associations were determined with Spearman correlation coefficients. We used the Mann-Whitney test to evaluate sex differences in CV risk variables (Table 1). Linear regression analyses were used to determine the relationship between adiposity and log transformed hs-CRP, stratified by sex. Path analysis was performed (EB) using SAS proc CALIS to test whether hs-CRP mediated the relationship between adiposity and IR. In the final path model we adjusted for sex and Tanner stage, however, these variables did not reach statistical significance. Standardized path coefficients and t-values are provided; $\mathrm{t}$-values greater than 1.96 reflect $\mathrm{p}<0.05$. As the model was fully saturated, with degrees of freedom equal to zero, goodness-of-fit for the overall model could not be estimated. Descriptive fit indices, root mean square error of approximation (RMSEA) and comparative fit index (CFI), were acceptable. Per $\mathrm{Hu}$ and Bentler [12], RMSEA close to 0.06 and CFI close to 0.95 are suitable.

\section{Results}

The sample consisted of 78 males and 59 females. Data related to obesity and CV risk factors are shown in Table 1. Adiposity was related to inflammation (log hs-CRP), WC for males $\left(\beta=0.36, \mathrm{p}<0.01, \mathrm{R}^{2}=0.13\right)$ and FMI for females $\left(\beta=0.37, \mathrm{p}<0.01, \mathrm{R}^{2}=0.14\right)$. Hs-CRP was associated with HOMA-IR in males and females $(\mathrm{p}<$ 0.05 ), independent of adiposity. In our path analysis, hsCRP partially mediated the relationship between adiposity and HOMA-IR, controlling for sex and Tanner stage ( $\mathrm{p}<$ 0.05 ) (Figure 1). For the entire sample, significant paths were found using either measure of adiposity. However, the model using WC as the measure of adiposity explained more of the variance in HOMA-IR than that using FMI.

\section{Discussion}

In our sample of overweight and obese Chilean adolescents, we found that inflammation significantly mediated the relationship between two measures of adiposity, WC and FMI, and increasing levels of HOMA-IR. The association between adiposity and hs-CRP has been extensively studied $[13,14]$. Likewise, inflammation has been associated

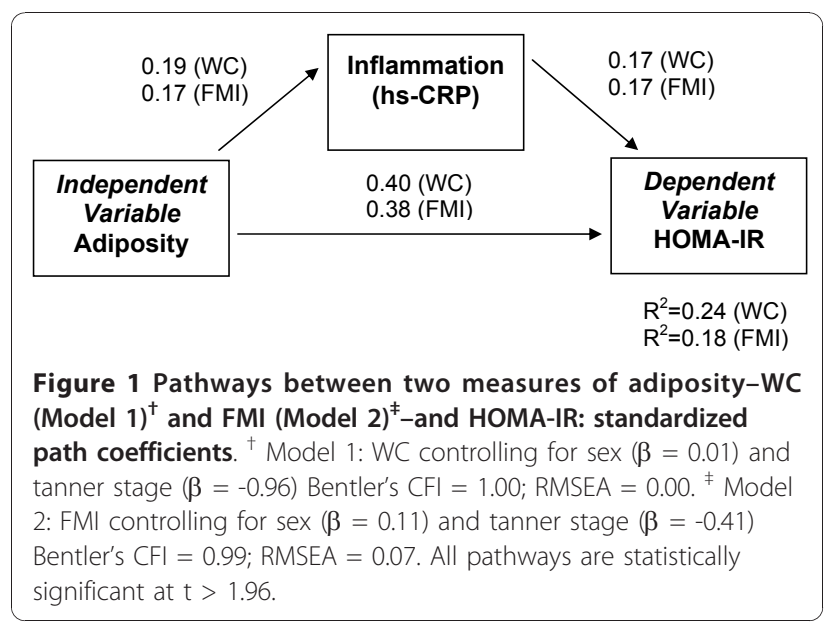

with IR [15]. However to our knowledge, there is no other human study showing inflammation as a potential pathophysiologic link between obesity and metabolic derangement, an association well-described in cell-culture and in animal models [16,17].

Gender differences in the relationship between adiposity and inflammation have been previously described in adults with waist-to-hip ratio related to inflammation in men and FM having a stronger association with inflammation among women [6]. Our results make an important contribution, as they indicate that gender differences, described in adults, may also occur in adolescents. During adolescence important sex differences in body composition emerge. Females have more peripheral fat; therefore, WC may not best reflect total fat. On the other hand, males have lower FM, but are likely to have more visceral fat [18]. Measures of adiposity are imperfect and are differentially related to visceral fat. Thus, it is important to choose the measure of adiposity most associated with later adverse outcomes.

Several limitations should be noted. Our findings cannot be generalized to normal weight adolescents or to adolescents from other backgrounds (not Chilean). Future studies should include more direct measures of visceral and subcutaneous fat content (e.g. magnetic resonance or ultrasound imaging), and additional measures of inflammatory status (e.g. interleukin 6, tumor necrosis factor alpha, adiponectin) and insulin sensitivity. It would also be important to assess the magnitude of other factors that might be related to inflammatory status and metabolic performance, for example nonalcoholic steatohepatitis which often occurs with obesity and IR and can progress to inflammation and fibrosis $[19,20]$. The strengths of the study include careful anthropometric assessment and reliable measurement of FM. Moreover, our subjects were recruited from a community population, rather than using a clinical sample. 
In conclusion, our sample of overweight and obese Chilean adolescents showed gender-specific associations between adiposity and systemic inflammation. Additionally, we found that hs-CRP statistically mediated the association between adiposity and IR. This suggests that a systemic inflammatory state is initiated by FM excess even during adolescence and that inflammation influences the metabolic consequences of overweight and obesity. Future research should include 1) detailed assessment of adiposity topography, 2) additional assessments of inflammation (e.g., inflammatory infiltration of adipose tissue) and 3) studies in other populations.

\section{Abbreviations}

BMl: body mass index; CFI: comparative fit index; CV: cardiovascular; FM: fat mass; FMI: fat mass index; HOMA-IR: homeostatic model assessment of insulin resistance; Hs-CRP: high-sensitivity C-reactive protein; INTA: Institute of Nutrition and Food Technology; IR: insulin resistance; RMSEA: root mean square error of approximation; WC: waist circumference.

\section{Acknowledgements}

This study was supported by the University of Chile: DID SAL02/12-2 (PI: Burrows) and NIH/NHLBI R01 HL088530 (PI: Gahagan).

\section{Author details}

${ }^{1}$ Institute of Nutrition and Food Technology (INTA), University of Chile, Santiago, Chile. ${ }^{2}$ Division of Child Development and Community Health, Department of Pediatrics, University of California, San Diego, USA. ${ }^{3}$ Center for Human Growth and Development, University of Michigan, Ann Arbor, USA

\section{Authors' contributions}

MR participated in the design of the manuscript, data analysis, helped to draft the manuscript and significantly reviewed the manuscript before submission. SG participated in the design of the manuscript, data analysis, helped to draft the manuscript and significantly reviewed the manuscript before submission. ED participated in the data acquisition and interpretation and reviewed the manuscript before submission. EB participated in the design of the manuscript, data analysis, helped to draft the manuscript and significantly reviewed the manuscript before submission. $L L$ participated in the data acquisition and analysis and reviewed the manuscript before submission. LL participated in the analysis and reviewed the manuscript before submission. RB participated in the data acquisition, analysis and interpretation, conceived the idea of the manuscript and reviewed the manuscript before submission. All authors read and approved the final manuscript.

\section{Competing interests}

The authors declare that they have no competing interests.

Received: 8 September 2010 Accepted: 14 January 2011 Published: 14 January 2011

\section{References}

1. Cook S, Auinger P, Li C, Ford ES: Metabolic syndrome rates in United States adolescents, from the National Health and Nutrition Examination Survey, 1999-2002. J Pediatr 2008, 152(2):165-170.

2. Steinberger J, Daniels SR: Obesity, insulin resistance, diabetes, and cardiovascular risk in children: an American Heart Association scientific statement from the Atherosclerosis, Hypertension, and Obesity in the Young Committee (Council on Cardiovascular Disease in the Young) and the Diabetes Committee (Council on Nutrition, Physical Activity, and Metabolism). Circulation 2003, 107(10):1448-1453.

3. Jensen MD: Is visceral fat involved in the pathogenesis of the metabolic syndrome? Human model. Obesity (Silver Spring) 2006, 14(Suppl 1):20S-24S.
4. Hotamisligil GS: Inflammation and metabolic disorders. Nature 2006, 444(7121):860-867

5. Cartier A, Cote M, Lemieux I, Perusse L, Tremblay A, Bouchard C, Despres JP: Sex differences in inflammatory markers: what is the contribution of visceral adiposity? Am J Clin Nutr 2009, 89(5):1307-1314

6. Thorand B, Baumert J, Doring A, Herder C, Kolb H, Rathmann W, Giani G, Koenig W: Sex differences in the relation of body composition to markers of inflammation. Atherosclerosis 2006, 184(1):216-224.

7. Fernandez JR, Redden DT, Pietrobelli A, Allison DB: Waist circumference percentiles in nationally representative samples of African-American, European-American, and Mexican-American children and adolescents. $J$ Pediatr 2004, 145(4):439-444.

8. Kuczmarski RJ, Ogden CL, Grummer-Strawn LM, Flegal KM, Guo SS, Wei R, Mei Z, Curtin LR, Roche AF, Johnson CL: CDC growth charts: United States. Adv Data 2000, 314:1-27.

9. Update on the 1987 Task Force Report on High Blood Pressure in Children and Adolescents: a working group report from the National High Blood Pressure Education Program. National High Blood Pressure Education Program Working Group on Hypertension Control in Children and Adolescents. Pediatrics 1996, 98(4 Pt 1):649-658.

10. Schoeller DA: Hydrometry. In Human body composition. Edited by: Roche A, Heymsfield S, Lohman TG. New York: Human Kinetics; 1996:25-43.

11. Van Itallie TB, Yang MU, Heymsfield SB, Funk RC, Boileau RA: Heightnormalized indices of the body's fat-free mass and fat mass: potentially useful indicators of nutritional status. Am J Clin Nutr 1990, 52(6):953-959.

12. Hu L, Bentler P: Cutoff criteria for fit indexes in covariance structure analysis: Conventional criteria versus new alternatives. Structural Equation Modeling 1999, 6:1-55.

13. Visser M, Bouter LM, McQuillan GM, Wener MH, Harris TB: Elevated Creactive protein levels in overweight and obese adults. JAMA 1999, 282(22):2131-2135.

14. Warnberg J, Nova E, Moreno LA, Romeo J, Mesana MI, Ruiz JR, Ortega FB, Sjostrom M, Bueno M, Marcos A: Inflammatory proteins are related to total and abdominal adiposity in a healthy adolescent population: the AVENA Study. Am J Clin Nutr 2006, 84(3):505-512.

15. Park K, Steffes M, Lee DH, Himes JH, Jacobs DR Jr: Association of inflammation with worsening HOMA-insulin resistance. Diabetologia 2009, 52(11):2337-2344.

16. Hotamisligil GS, Murray DL, Choy LN, Spiegelman BM: Tumor necrosis factor alpha inhibits signaling from the insulin receptor. Proc Natl Acad Sci USA 1994, 91(11):4854-4858.

17. Weisberg SP, Hunter D, Huber R, Lemieux J, Slaymaker S, Vaddi K, Charo I, Leibel RL, Ferrante AW Jr: CCR2 modulates inflammatory and metabolic effects of high-fat feeding. J Clin Invest 2006, 116(1):115-124.

18. Wells JC: Sexual dimorphism of body composition. Best Pract Res Clin Endocrinol Metab 2007, 21(3):415-430.

19. Tarantino G, Colicchio P, Conca P, Finelli C, Di Minno MN, Tarantino M, Capone D, Pasanisi F: Young adult obese subjects with and without insulin resistance: what is the role of chronic inflammation and how to weigh it non-invasively? J Inflamm 2009, 16(6):1-6.

20. Patton HM, Yates K, Unalp-Arida A, Behling CA, Huang TT, Rosenthal $P$, Sanyal AJ, Schwimmer JB, Lavine JE: Association between metabolic syndrome and liver histology among children with nonalcoholic Fatty liver disease. Am J Gastroenterol 2010, 105(9):2093-102.

doi:10.1186/1475-2891-10-4

Cite this article as: Reyes et al:: Relationship of Adiposity and Insulin Resistance Mediated by Inflammation in a Group of Overweight and Obese Chilean Adolescents. Nutrition Journal 2011 10:4 